\title{
BALANCING CONDITIONS OF PLANAR MECHANISMS WITH MULTI-DEGREE OF FREEDOM
}

\author{
Nguyen Van Khang, Nguyen Phong Dien, Pham Van Son \\ Hanoi University of Technology
}

\begin{abstract}
This paper presents a new method for deriving the balancing conditions of planar mechanisms with multi-degree of freedom. The method has advantage of being suitable for the application of the widely accessible computer algebra systems such as MAPLE. In the example, the conditions for complete shaking force and shaking moment balancing of a planar five-bar linkage are given.
\end{abstract}

\section{INTRODUCTION}

One of the problems of the complete shaking force and shaking moment balancing of planar mechanism consist of the deriving the so-called balancing conditions. These balancing conditions will be used to determine the size and location of counterweights or supplementary links which must be added to the initial mechanism, in order to eliminate the shaking force and the shaking moment caused by all moving links. The balancing methods for planar mechanisms with one degree-of-freedom can be found in a large number of publications [2-7]. However, the development on the balancing theory of planar mechanisms with multi-degree of freedom is still limited and the literature on this respect therefore is very little.

In the paper, we presents a new method for deriving the general balancing conditions of planar mechanisms with multi-degree of freedom and an arbitrary structure. The developed algorithm is suitable for the application of the widely accessible computer algebra systems such as MAPLE. In the following example, the conditions for complete shaking force and shaking moment balancing of a planar five-bar linkage are given.

\section{BALANCING THEORY OF PLANAR MECHANISMS WITH MULTI-DEGREE OF FREEDOM}

We first consider an arbitrary link of a planar mechanism with multi-degree-of-freedom shown in Fig. 1. The mechanism consist of a set of $n$ links in a closed loop structure with holonomic and rheonomic constraints.

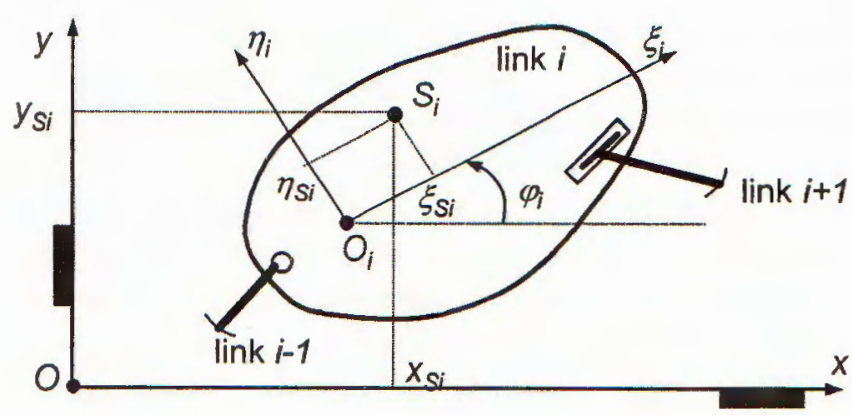

Fig. 1. Definition of coordinates 
The shaking force and the shaking moment transmitted to the ground link 1 from all moving links can be expressed in the form [1, 8]:

$$
\begin{aligned}
\boldsymbol{F}^{*} & =-\frac{d}{d t} \boldsymbol{p}, \\
M_{O}^{*} & =-\frac{d}{d t} \boldsymbol{L}_{O},
\end{aligned}
$$

where $\mathbf{p}$ is the resultant linear momentum and $\mathbf{L}_{O}$ the resultant angular momentum of the mechanism taken about fixed point $O$. Assuming that only planar linkages are considered, three components of the linear momentum $\mathbf{p}$ and the angular momentum $\mathbf{L}_{O}$ are given by

$$
\begin{aligned}
& \boldsymbol{p}_{x}=\sum_{i=2}^{n} m_{i} \dot{x}_{S i}, \boldsymbol{p}_{y}=\sum_{i=2}^{n} m_{i} \dot{y}_{S i}, \boldsymbol{p}_{z}=0 \\
& \boldsymbol{L}_{O x}=\boldsymbol{L}_{O y}=0, \quad \boldsymbol{L}_{O z}=\sum_{i=2}^{n}\left[m_{i}\left(x_{S i} \dot{y}_{S i}-y_{S i} \dot{x}_{S i}\right)+J_{S i} \dot{\varphi}_{i}\right]
\end{aligned}
$$

where $m_{i}$ is the mass of the $i$-th link and $J_{S i}$ represents the moment of inertia of the link about the axis passing through the center of mass $S_{i}$ and perpendicular to the plane of motion. Now we choose $f$ generalized coordinates $q=\left[q_{1}, q_{2}, \ldots, q_{f}\right]^{T}$ corresponding to the $f$ degrees of freedom of the system, the velocity of the center of mass $S_{i}$ and the angular velocity $\dot{\varphi}_{i}$ can be written as

$$
\dot{x}_{S i}=\frac{\partial x_{S i}}{\partial q} \dot{q}, \dot{y}_{S i}=\frac{\partial y_{S i}}{\partial \boldsymbol{q}} \dot{\boldsymbol{q}}, \dot{\varphi}_{S i}=\frac{\partial \varphi_{i}}{\partial \boldsymbol{q}} \dot{\boldsymbol{q}} .
$$

Substituting (2.3), (2.4) and (2.5) into equations (2.1) and (2.2), three components of the shaking force $F^{*}$ and the shaking moment $M_{O}^{*}$ can be written in the form

$$
\begin{aligned}
& F_{x}^{*}=-\frac{d}{d t} \sum_{i=2}^{n}\left(m_{i} \frac{\partial x_{S i}}{\partial \boldsymbol{q}}\right) \dot{\boldsymbol{q}}, \quad F_{y}^{*}=-\frac{d}{d t} \sum_{i=2}^{n}\left(m_{i} \frac{\partial y_{S i}}{\partial \boldsymbol{q}}\right) \dot{\boldsymbol{q}}, \quad F_{z}^{*}=0, \\
& M_{O x}^{*}=M_{O y}^{*}=0, \quad M_{O z}^{*}=-\frac{d}{d t} \sum_{i=2}^{n}\left(m_{i} x_{S i} \frac{\partial y_{S i}}{\partial \boldsymbol{q}}-y_{S i} \frac{\partial x_{S i}}{\partial \boldsymbol{q}}+J_{S i} \frac{\partial \varphi_{i}}{\partial q}\right) \dot{q} .
\end{aligned}
$$

So, the planar mechanism can be completely balanced if the shaking force and the shaking moment vanish, this yields

$$
\begin{aligned}
& \sum_{i=2}^{n} m_{i} x_{S i, i}=0, \quad j=1,2, \ldots, f \\
& \sum_{i=2}^{n} m_{i} y_{S i, j}=0, \quad j=1,2, \ldots, f, \\
& \sum_{i=2}^{n} m_{i}\left(x_{S i} y_{S i, j}-y_{S i} x_{S i, j}\right)+J_{S i} \varphi_{i, j}=0, \quad j=1,2, \ldots, f,
\end{aligned}
$$


where $x_{S i, j}=\frac{\partial x_{S i}}{\partial q_{j}}, \quad y_{S i, j}=\frac{\partial y_{S i}}{\partial q_{j}}, \varphi_{i, j}=\frac{\partial \varphi_{i}}{\partial q_{j}}$.

Equations (2.8), (2.9) and (2.10) are the general conditions for complete balancing of $a$ arbitrary planar mechanism with multi-degree of freedom.

By introducing the new vector $\mathbf{z}$ which contains functions of the variables $q_{j}$, the coordinates of the center of mass $S_{i}$ in the fixed coordinate frame $\{x, y\}$ can be expressed as [3].

$$
\begin{aligned}
& x_{S i}=e_{x i}^{*}+\boldsymbol{a}_{i}^{T} z, \\
& y_{S i}=\boldsymbol{e}_{y i}^{*}+\boldsymbol{b}_{i}^{T} \boldsymbol{z}, \quad i=2,3, \ldots, n,
\end{aligned}
$$

where the vectors $\mathbf{a}_{i}$ and $\mathbf{b}_{i}$ contain components which are independent of $\mathbf{q}, e_{x i}^{*}$ and $e_{y i}^{*}$ are constant parameters. Analog to the equations (2.11), the kinematic relationships for the links of the mechanism (i.e. loop equations) may be written in the form

$$
C z=f, \quad C=\left[C_{I}, C_{I I}\right] .
$$

Here the matrix $\mathbf{C}$ and the vector $\mathbf{f}$ include the components which are geometrical parameters and independent of $\mathbf{q}$. A partitioning of vector $\mathbf{z}$ from equation (2.12)

$$
z=\left[\begin{array}{c}
v \\
w
\end{array}\right]
$$

leads to the following relation

$$
C_{I} v+C_{I I} w=f .
$$

The matrix $\mathbf{C}_{I I}$ is chosen so that it must be a square matrix and nonsingular. The dimension of vector $\mathbf{w}$ and the number of the loop equations are equal. By solving (2.14) with the vector of variables $w$, we have

$$
w=C_{I I}^{-1}\left(f-C_{I} v\right) .
$$

By using equations (2.13) and (2.15), the coordinates of the center of mass $S_{i}$ and their partial derivatives can be expressed in terms of the reduced vector of variables $\mathbf{v}$ as

$$
\begin{aligned}
& x_{S i}=e_{x i}+\boldsymbol{g}_{i}^{T} \boldsymbol{v}, \quad y_{S i}=e_{y i}+\boldsymbol{h}_{i}^{T} \boldsymbol{v}, \\
& x_{S i, j}=\boldsymbol{g}_{i}^{T} \frac{\partial \boldsymbol{v}}{\partial q_{j}}, y_{S i, j}=\boldsymbol{h}_{i}^{T} \frac{\partial v}{\partial q_{j}}
\end{aligned}
$$

where

$$
\begin{aligned}
& g_{i}^{T}=a_{i I}^{T}-a_{i I I}^{T} C_{I I}^{-1} C_{I}, \quad h_{i}^{T}=b_{i I}^{T}-b_{i I}^{T} C_{I I}^{-1} C_{I}, \\
& e_{x i}=e_{x i}^{*}+a_{i I I}^{T} C_{I I}^{-1} f, \quad e_{y i}=e_{y i}^{*}+b_{i I I}^{T} C_{I I}^{-1} f .
\end{aligned}
$$

Substituting equation (2.17) into balancing conditions (2.8) and (2.9), we obtain

$$
\left(\sum_{i=2}^{n} m_{i} g_{i}^{T}\right) \frac{\partial v}{\partial q_{j}}=0,\left(\sum_{i=2}^{n} m_{i} h_{i}^{T}\right) \frac{\partial v}{\partial q_{j}}=0 .
$$


Finally, the conditions for complete shaking force balancing take the compact form

$$
\sum_{i=2}^{n} m_{i} \boldsymbol{g}_{i}^{T}=0, \sum_{i=2}^{n} m_{i} \boldsymbol{h}_{i}^{T}=0
$$

In order to extract the conditions for the shaking moment balancing, some additional transformations are required. Substituting (2.16) and (2.17) into equation (2.10), we have

$$
\left[\sum_{i=2}^{n} m_{i}\left(e_{x i} \boldsymbol{h}_{i}^{T}-e_{y i} \boldsymbol{g}_{i}^{T}\right)\right] \frac{\partial v}{\partial q_{j}}+v^{T}\left[\sum_{i=2}^{n} m_{i}\left(g_{i} \boldsymbol{h}_{i}^{T}-\boldsymbol{h}_{i} \boldsymbol{g}_{i}^{T}\right)\right] \frac{\partial v}{\partial q_{j}}+\sum_{i=2}^{n} J_{S i} \varphi_{i, j}=0 .
$$

By introducing the vector $\mathbf{u}$

$$
\boldsymbol{u}=\sum_{i=2}^{n} m_{i}\left(e_{x i} \boldsymbol{h}_{i}^{T}-e_{y i} \boldsymbol{g}_{i}^{T}\right)
$$

and the matrix $\mathbf{S}$

$$
\boldsymbol{S}=\sum_{i=2}^{n} m_{i}\left(g_{i} \boldsymbol{h}_{i}^{T}-\boldsymbol{h}_{i} \boldsymbol{g}_{i}^{T}\right)
$$

Equation (2.22) takes the form

$$
u^{T} \frac{\partial v}{\partial q_{j}}+v^{T} S \frac{\partial v}{\partial q_{j}}+\sum_{i=2}^{n} J_{S i} \varphi_{i, j}=0
$$

If the vector $\mathbf{z}$ has the following form

$$
z=\left[\cos \varphi_{2}, \cos \varphi_{3}, \ldots, \cos \varphi_{n}, \sin \varphi_{2}, \sin \varphi_{3}, \ldots, \sin \varphi_{n}\right]^{T},
$$

the partial derivatives $\varphi_{i, j}$ can be expressed as

$$
\varphi_{i, j}=z_{i-1} z_{(n+i-2), j}-z_{n+i-2} z_{(i-1), j}, i=2,3, \ldots, n .
$$

As can be seen from equation (2.27), the third term in equation (2.25) can be written in the matrix form as

$$
\sum_{i=2}^{n} J_{S i} \varphi_{i, j}=z^{T} D \frac{\partial z}{\partial q_{j}}
$$

where the matrix $\mathbf{D}$ contains the components which are independent of $q$. The matrix $\mathbf{D}$ can be partitioned in four sub-matrices corresponding to the vector of variables $\mathbf{v}$ und $\mathbf{w}$ as follows

$$
D=\left[\begin{array}{ll}
D_{11} & D_{12} \\
D_{21} & D_{22}
\end{array}\right]
$$

By using the relation (2.15) and (2.29), the following relation is found from equation (2.28)

$$
\begin{aligned}
& \boldsymbol{z}^{T} \boldsymbol{D} \frac{\partial \boldsymbol{z}}{\partial q_{j}}=\left[\begin{array}{c}
\boldsymbol{v} \\
w
\end{array}\right]^{T}\left[\begin{array}{ll}
D_{11} & D_{12} \\
D_{21} & D_{22}
\end{array}\right]\left[\begin{array}{c}
\frac{\partial v}{\partial q_{j}} \\
\frac{\partial w}{\partial q_{j}}
\end{array}\right]= \\
& v^{T}\left[D_{11}+\left(C_{I I}^{-1} C_{I}\right)^{T}\left(D_{22} C_{I I}^{-1} C_{I}-D_{21}\right)-D_{12} C_{I I}^{-1} C_{I}\right] \frac{\partial v}{\partial q_{j}}+ \\
& +\left(C_{I I}^{-1} f\right)^{T}\left(D_{21}-D_{22} C_{I I}^{-1} C_{I}\right) \frac{\partial v}{\partial q_{j}}
\end{aligned}
$$


By introducing the vector $u^{*}$

$$
u^{*}=\left(C_{I I}^{-1} f\right)^{T}\left(D_{21}-D_{22} C_{I I I}^{-1}\right)
$$

and the matrix $S^{*}$

$$
S^{*}=D_{11}+\left(C_{I I}^{-1} C_{I}\right)^{T}\left(D_{22} C_{I I}^{-1} C_{I}-D_{21}\right)-D_{12} C_{I I}^{-1} C_{I},
$$

equation (2.25) takes the form

$$
\left(\boldsymbol{u}+\boldsymbol{u}^{*}\right)^{T} \frac{\partial v}{\partial q_{j}}+v^{T}\left(S+S^{*}\right) \frac{\partial v}{\partial q_{j}}=0 .
$$

Finally, the following conditions for complete shaking moment balancing are found from equation (2.33)

$$
u+u^{*}=0, \quad S+S^{*}=0 .
$$

The described algorithm allows to derive the balancing conditions (2.21) and (2.34) for planar mechanisms with multi-degree of freedom systematically.

\section{EXAMPLE}

In the following example we introduce the application of the balancing theory described above to a planar five-bar linkage with two degrees of freedom which is shown in Fig. 2. Five revolute pairs are required to connect the five links. The driving links are termed as link 2 and link 5 which are hinged to the ground link 1.

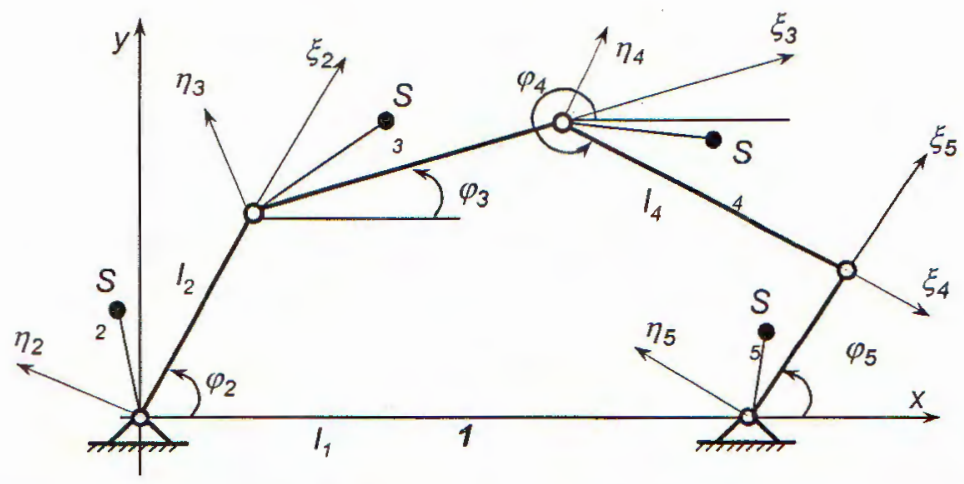

Fig. 2. A planar five-bar linkage

First, we introduce the fixed coordinate frame $\{x, y\}$ and the moving coordinate frame $\left\{\xi_{i}, \eta_{i}\right\}$ as shown in Fig. 2. The configuration of the manipulator is also prescribed by rotation angles $\varphi_{2}, \varphi_{3}, \varphi_{4}$ and $\varphi_{5}$. The independent generalized coordinates $q_{1}=$ $\varphi_{2}, q_{2}=\varphi_{5}$ are chosen. The loop equations of the mechanism can be expressed in the form

$$
\begin{aligned}
& l_{2} \cos \varphi_{2}+l_{3} \cos \varphi_{3}+l_{4} \cos \varphi_{4}-l_{5} \cos \varphi_{5}=l_{1}, \\
& l_{2} \sin \varphi_{2}+l_{3} \sin \varphi_{3}+l_{4} \sin \varphi_{4}-l_{5} \sin \varphi_{5}=0,
\end{aligned}
$$


where $l_{i}$ denotes the length of the $i$-th link. According to equation (2.26) we choose the vector $\mathbf{z}$ with the following form

$$
z=\left[\cos \varphi_{2}, \cos \varphi_{3}, \cos \varphi_{4}, \cos \varphi_{5}, \sin \varphi_{2}, \sin \varphi_{3}, \sin \varphi_{4}, \sin \varphi_{5}\right]^{T} .
$$

The coordinates of the center of mass $S_{i}(i=2,3,4,5)$ in the fixed coordinate $\{x, y\}$ are expressed in term of the vector $\mathbf{z}$ as

$$
\begin{aligned}
& x_{S 2}=\left[\begin{array}{llllllll}
\xi_{S 2} & 0 & 0 & 0 & -\eta_{S 2} & 0 & 0 & 0
\end{array}\right] \boldsymbol{z}, \\
& y_{S 2}=\left[\begin{array}{lllllllll}
\eta_{S 2} & 0 & 0 & 0 & \xi_{S 2} & 0 & 0 & 0
\end{array}\right] z, \\
& x_{S 3}=\left[\begin{array}{lllllllll}
l_{2} & \xi_{S 3} & 0 & 0 & 0 & -\eta_{S 3} & 0 & 0
\end{array}\right] z, \\
& y_{S 3}=\left[\begin{array}{lllllllll}
0 & \eta_{S 3} & 0 & 0 & l_{2} & \xi_{S 3} & 0 & 0
\end{array}\right] \boldsymbol{z}, \\
& x_{S 4}=\left[\begin{array}{llllllll}
l_{2} & l_{3} & \xi_{S 4} & 0 & 0 & 0 & -\eta_{S 4} & 0
\end{array}\right] \boldsymbol{z}, \\
& \left.y_{S 4}=0 \begin{array}{llllllll}
\eta_{S 4} & 0 & l_{2} & l_{3} & \xi_{S 4} & 0
\end{array}\right] \boldsymbol{z} \\
& x_{S 5}=l_{1}+\left[\begin{array}{lllllllll}
0 & 0 & 0 & \xi_{S 5} & 0 & 0 & 0 & -\eta_{S 5}
\end{array}\right] \boldsymbol{z}, \\
& y_{S 5}=\left[\begin{array}{lllllllll}
0 & 0 & 0 & \eta_{S 5} & 0 & 0 & 0 & \xi_{S 5}
\end{array}\right] \boldsymbol{z},
\end{aligned}
$$

where $\xi_{S i}, \eta_{S i}$ are coordinates of $S_{i}$ in the moving coordinate frame $\left\{\xi_{i}, \eta_{i}\right\}$. It can be shown that the loop equations in (3.1) have the form

$$
\left[\begin{array}{llllllll}
l_{2} & l_{3} & l_{4} & -l_{5} & 0 & 0 & 0 & 0 \\
0 & 0 & 0 & 0 & l_{2} & l_{3} & l_{4} & -l_{5}
\end{array}\right] z=\left[\begin{array}{c}
l_{1} \\
0
\end{array}\right]
$$

The reduced vector of variables $\mathbf{v}$ and the vector of eliminated variables $\mathbf{w}$ are selected from the original vector $\mathrm{z}$ as follows

$$
\begin{gathered}
v=\left[\cos \varphi_{2}, \cos \varphi_{3}, \cos \varphi_{5}, \sin \varphi_{2}, \sin \varphi_{3}, \sin \varphi_{5}\right]^{T}, \\
w=\left[\cos \varphi_{4}, \sin \varphi_{4}\right]^{T} .
\end{gathered}
$$

So, the matrix $\mathbf{C}_{I}, C_{I I}^{-1}$ and the vector $\mathbf{f}$ described in equation (2.14) can be determined

$$
C_{I}=\left[\begin{array}{cccccc}
l_{2} & l_{3} & -l_{5} & 0 & 0 & 0 \\
0 & 0 & 0 & l_{2} & l_{3} & -l_{5}
\end{array}\right], C_{I I}^{-1}=\frac{1}{l_{4}}\left[\begin{array}{ll}
1 & 0 \\
0 & 1
\end{array}\right], f=\left[\begin{array}{c}
l_{1} \\
0
\end{array}\right]
$$

With the known coordinates of the center of masses and the matrix $\mathrm{C}_{I}, C_{I I}^{-1}$, the vectors $\mathbf{g}_{i}$ and $\mathbf{h}_{i}$ can be determined according to equation (2.18) without any difficulty. Then, by substituting all these results into equations (2.21), we find six conditions for the complete shaking force balancing

$$
\begin{aligned}
& m_{2} l_{4} \eta_{S 2}-m_{4} l_{2} \eta_{S 4}=0 \\
& m_{3} l_{4} \eta_{S 3}-m_{4} l_{3} \eta_{S 4}=0 \\
& m_{4} l_{5} \eta_{S 4}+m_{5} l_{4} \eta_{S 5}=0 \\
& m_{3} l_{4} \xi_{S 3}+m_{4} l_{3}\left(l_{4}-\xi_{S 4}\right)=0 \\
& m_{4} l_{5} \xi_{S 4}+m_{5} l_{4} \xi_{S 5}=0 \\
& m_{2} l_{4} \xi_{S 2}+m_{3} l_{4} l_{2}+m_{4} l_{2}\left(l_{4}-\xi_{S 4}\right)=0 .
\end{aligned}
$$


For the purpose of the shaking moment balancing, four additional links are added to the initial mechanism (see Fig. 3). Note that they have no influence on the kinematic function of the initial mechanism. The basis of this solution was proposed in several previous studies $[2],[3]$.

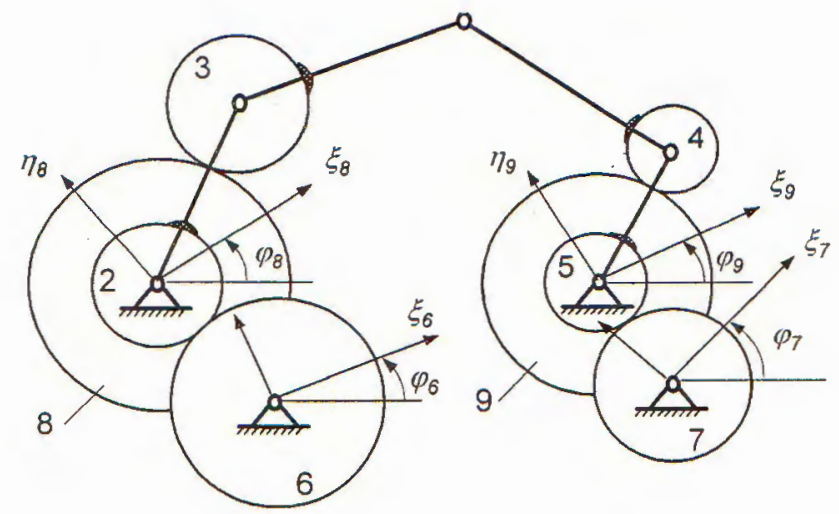

Fig. 3. The planar five-bar linkage with four supplementary gear-pairs for complete shaking moment balancing

Assumed that the mass centers of the gears 6, 7, 8 and 9 are located on the rotating axes (i.e. $\xi_{S 6}=\xi_{S 7}=\xi_{S 8}=\xi_{S 9}=0, \eta_{S 6}=\eta_{S 7}=\eta_{S 8}=\eta_{S 9}=0$ ). While maintaining the shaking force balancing, the supplementary gear-pairs create additional balancing moments, thereby eliminating the shaking moment. According to Fig. 3, the constraints for the gear-pairs are

$$
\begin{aligned}
& r_{2} \varphi_{2, j}+r_{6} \varphi_{6, j}=0, \\
& r_{5} \varphi_{5, j}+r_{7} \varphi_{7, j}=0 \\
& r_{3} \varphi_{3, j}+r_{8} \varphi_{8, j}-\left(r_{3}+r_{8}\right) \varphi_{2, j}=0, \\
& r_{4} \varphi_{4, j}+r_{9} \varphi_{9, j}-\left(r_{4}+r_{9}\right) \varphi_{5, j}=0,
\end{aligned}
$$

where $r_{i}$ is the radius of $i$-th gear and $j=1,2$. Based on equation (2.28) and the vector $\mathbf{z}$ given from (3.2) we can determine the matrix $\mathbf{D}=\left[d_{m n}\right]$ as follows

$$
\begin{aligned}
& d_{15}=-d_{51}=J_{S 2}-\frac{r_{2}}{r_{6}} J_{S 6}+\left(1+\frac{r_{3}}{r_{8}}\right) J_{S 8}, \\
& d_{26}=-d_{62}=J_{S 3}-\frac{r_{3}}{r_{8}} J_{S 8}, \\
& d_{37}=-d_{73}=J_{S 4}-\frac{r_{4}}{r_{9}} J_{S 9}, \\
& d_{48}=-d_{84}=J_{S 5}-\frac{r_{5}}{r_{7}} J_{S 7}+\left(1+\frac{r_{4}}{r_{9}}\right) J_{S 9}
\end{aligned}
$$

and every other $d_{m n}=0$.

By partitioning of the matrix $\mathbf{D}$ related to equation (2.29), we obtain the submatrices $\mathbf{D}_{11}, \mathbf{D}_{12}, \mathbf{D}_{21}, \mathbf{D}_{22}$. Then, the vectors $\mathbf{u}$ and $u^{*}$, the matrices $\mathbf{S}$ and $S^{*}$ are formulated by using equations (2.23), (2.24), (2.31) and (2.32). Finally, according to equation (2.34) 
we find the following balancing conditions of the shaking moment for the mechanism with " additional links

$$
\begin{aligned}
& m_{3} l_{2} \eta_{S 3}=0, \\
& m_{4} l_{5} \eta_{S 4}=0 \\
& m_{5} l_{1} \eta_{S 5}=0 \\
& J_{S 2}-\frac{r_{2}}{r_{6}} J_{S 6}+\left(1+\frac{r_{3}}{r_{8}}\right) J_{S 8}+\left(\frac{l_{2}}{l_{4}}\right)^{2}\left(J_{S 4}-\frac{r_{4}}{r_{9}} J_{S 9}\right)+m_{2}\left(\xi_{S 2}^{2}+\eta_{S 2}^{2}\right) \\
& +\left(m_{3}+m_{4}\right) l_{2}^{2}+m_{4}\left(\frac{l_{2}}{l_{4}}\right)^{2}\left(\xi_{S 4}^{2}+\eta_{S 4}^{2}\right)-2\left(\frac{l_{2}}{l_{4}}\right) m_{4} l_{2} \xi_{S 4}=0, \\
& J_{S 4}-\frac{r_{4}}{r_{9}} J_{S 9}+\left(\frac{l_{4}}{l_{3}}\right) m_{3} l_{4} \xi_{S 3}+m_{4}\left(\xi_{S 4}^{2}+\eta_{S 4}^{2}\right)+m_{4}\left(l_{4}^{2}-2 l_{4} \xi_{S 4}\right)=0, \\
& J_{S 4}-\frac{r_{4}}{r_{9}} J_{S 9}+m_{4}\left(\xi_{S 4}^{2}+\eta_{S 4}^{2}\right)-m_{4} l_{4} \xi_{S 4}=0, \\
& J_{S 3}-\frac{r_{3}}{r_{8}} J_{S 8}+\left(\frac{l_{3}}{l_{4}}\right)^{2}\left(J_{S 4}-\frac{r_{4}}{r_{9}} J_{S 9}\right)+m_{3}\left(\xi_{S 3}^{2}+\eta_{S 3}^{2}\right) \\
& +m_{4} l_{3}^{2}+m_{4}\left(\frac{l_{3}}{l_{4}}\right)^{2}\left(\xi_{S 4}^{2}+\eta_{S 4}^{2}\right)-2\left(\frac{l_{3}}{l_{4}}\right) m_{4} l_{3} \xi_{S 4}=0, \\
& J_{S 5}-\frac{r_{5}}{r_{7}} J_{S 7}+\left(1+\frac{r_{4}}{r_{9}}\right) J_{S 9}+\left(\frac{l_{5}}{l_{4}}\right)^{2}\left(J_{S 4}-\frac{r_{4}}{r_{9}} J_{9}\right) \\
& +\left(\frac{l_{5}}{l_{4}}\right)^{2} m_{4}\left(\xi_{S 4}^{2}+\eta_{S 4}^{2}\right)+m_{5}\left(\xi_{S 5}^{2}+\eta_{S 5}^{2}\right)=0 \\
& J_{S 4}-\frac{r_{4}}{r_{9}} J_{S 9}+m_{4}\left(\xi_{S 4}^{2}+\eta_{S 4}^{2}\right)+\frac{l_{4}}{l_{5}} m_{5} l_{4} \xi_{S 5}=0 .
\end{aligned}
$$

Furthermore, the above equations can be simplified by inserting (3.20) into the remaining equations. The obtained conditions become to be independent of $J_{S 9}$. After assigning $r_{9}=0$, we get the reduced conditions for the complete shaking moment balancing

$$
\begin{aligned}
& \eta_{S 3}=0, \quad \eta_{S 4}=0, \quad \eta_{S 5}=0 \\
& J_{S 2}-\frac{r_{2}}{r_{6}} J_{S 6}+\left(1+\frac{r_{3}}{r_{8}}\right) J_{S 8}+m_{2}\left(\xi_{S 2}^{2}+\eta_{S 2}^{2}\right)+\left(m_{3}+m_{4}\right) l_{2}^{2}+\left(\frac{l_{2}}{l_{4}}\right) m_{4} \xi_{S 4} l_{2}=0 \\
& m_{3} l_{4} \xi_{S 3}+m_{4} l_{3}\left(l_{4}-\xi_{S 4}\right)=0 \\
& J_{S 3}-\frac{r_{3}}{r_{8}} J_{S 8}+m_{3}\left(\xi_{S 3}^{2}+\eta_{S 3}^{2}\right)+\left(\frac{l_{3}}{l_{4}}\right)\left(m_{4} l_{4} l_{3}-m_{4} \xi_{S 4} l_{3}\right)=0 \\
& J_{S 4}+J_{S 5}-\frac{r_{5}}{r_{7}} J_{S 7}+m_{4}\left(\xi_{S 4}^{2}+\eta_{S 4}^{2}\right)-m_{4} \xi_{S 4} l_{4}+\left(\frac{l_{5}}{l_{4}}\right) m_{4} \xi_{S 4} l_{5}+m_{5}\left(\xi_{S 5}^{2}+\eta_{S 5}^{2}\right)=0 \\
& m_{4} l_{5} \xi_{S 4}+m_{5} l_{4} \xi_{S 5}=0
\end{aligned}
$$

\section{DISCUSSION AND CONCLUSIONS}

As it can be seen from the above obtained conditions, the shaking moment of the mechanism can be completely balanced without using the gear-pair (4) and (9). Note that conditions (3.24), (3.26), (3.29) of the shaking moment balancing are fulfilled with five conditions $(3.8)-(3.12)$ of the shaking force balancing. 
Conditions for the shaking force balancing (3.8) - (3.12) can be satisfied by internal mass redistribution or adding counterweights mounted on the links. These conditions will be used to determine the size and the location of the counterweights. In practice, conditions (3.25), (3.27), (3.28) may give the design guidelines for the shaking moment balancing of the mechanism with supplementary links .

The example has demonstrated that the proposed balancing theory can be appropriately used for deriving the balancing conditions of planar mechanisms with multi-degree of freedom. It is advantageous for application of the widely accessible computer algebra systems.

Acknowledgment. This paper was completed with the financial support of the Vietnam Basic Research Program in Natural Science

\section{REFERENCES}

1. Nguyen Van Khang, Über den Massenausgleich in Mehrkörpersystemen, Technische Mechanik, Band 14, Heft 3/4 (1994) 231-238.

2. H. Dresig, L. Rochausen, S. Naake, Balancing Conditions for planar Mechanism, ASME-Joumal: Flexible Mechanism, Dynamics and Analysis 47 (1992) 67-73.

3. H. Dresig, S. Naake, L. Rochausen, Vollständiger und harmonische Ausgleich ebener Mechanismen. Fortschritt-Berichte VDI, Reihe 18, Nr. 155, Düsseldorf: VDI Verlag 1994.

4. V. Arakelian, M. R. Smith, Shaking Moment Minimization of Fully Force-Balanced Linkages. Proc. of the $11^{\text {th }}$ World Congress in Mechanism and Machine Science, Tianjin, China, 2004.

5. I. S. Kochev, General Method for Active Balancing of Combined Shaking Moment and Torque Fluctuations in Planar Linkages, Mechanism and Machine Theory 25 (1990) 679-687.

6. G. G. Lawen, F. R. Tepper, R. S. Berkorf, Balancing of Linkages - An Update. Mechanism and Machine Theory 18 (1983) 213-220.

7. H. Dresig, I. I. Vulfson, Dynamik der Mechanismen, Springer Verlag, Wien, 1989.

8. W. Schiehlen, Technische Dynamik, B.G. Teubner, Stuttgart, 1986.

Received May 20, 2005

\section{CÁC DIỀU KIỆN CÂN BẰNG CƯA CƠ CẤU PHĂNG NHIỀU BẬC TỰ DO}

Bài báo này giới thiệu một phương pháp mới để thiết lập các điều kiện cân bằng của cơ cấu phẳng nhiều bậc tự do. Phương pháp này có ưu điểm là thích hợp với việc áp dụng các chương trình đại số Computer đang sử dụng rộng rãi như MAPLE. Các hệ thức cân băng lực quán tính và mô men lực quán tính của một cơ cấu phẳng năm khâu được trình bày trong một thí dụ áp dụng. 